\title{
Desigualdade no acesso aos Cuidados de Saúde Primários - a outra face das USF
}

Isabel Cortez*

\section{RESUMO}

Portugal é um país com profundas desigualdades económicas e sociais. A desigualdade no acesso aos Cuidados de Saúde Primários é uma das vertentes dessa desigualdade e não tem havido uma política eficaz para a atenuar. A afectação privilegiada de recursos materiais e humanos a determinados projectos tem, por vezes, agravado os cuidados noutros sectores, repetindo assim alguns dos erros das reformas do SNS britânico nos anos 90 .

Estima-se em mais de um milhão o número de utentes sem médico de família em Portugal, estando cerca de $40 \%$ localizados na área geográfica da Administração Regional de Saúde de Lisboa e Vale do Tejo. As Unidades de Saúde Familiar, apesar de serem uma peça importante na melhoria dos Cuidados de Saúde Primários, não vão dar resposta, a curto prazo, à falta de médicos de família. O mesmo se poderá dizer da contratação de médicos estrangeiros ou dos licenciados pelos novos cursos de Medicina.

Impõe-se, urgentemente, adoptar outro tipo de medidas para resolver a situação dos utentes sem médico de família. Alguns países europeus, confrontados com o mesmo problema, conseguiram uma solução de qualidade valorizando o papel dos enfermeiros, delegando-lhes várias áreas da prevenção e da vigilância de doenças crónicas. Isto permitiu o aumento da dimensão das listas dos médicos de família sem acréscimo relevante da carga de trabalho e mantendo a qualidade da prestação de cuidados.

Palavras-chave: Desigualdade; Acesso; Cuidados Saúde Primários.

\section{INTRODUÇÃO}

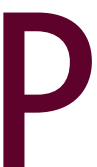

ortugal é um país com profundas desigualdades económicas e sociais, como tem sido frequentemente referido nos últimos anos, nomeadamente em vários discursos do Presidente da República. Estas desigualdades têm vindo a aumentar e Portugal tem-se mantido como o país da União Europeia com maior desigualdade em termos de rendimento, mesmo após o alargamento aos países de Leste. ${ }^{1,2}$

Frequentemente, os serviços de saúde reproduzem as assimetrias sociais. Os países com melhores resultados em saúde, nomeadamente os países escandinavos, são também os que apresentam menor desigualdade social e menor desigualdade na oferta de cuidados de saúde, ${ }^{1,3,4}$ pelo que o combate à desigualdade deveria ser uma prioridade política fundamental.

Os utentes sem médico de família (MF) constituem

*Assistente Graduada de Clínica Geral, UCSP Buraca, Amadora um grupo de maior vulnerabilidade em termos de saúde e são uma endemia silenciosa em alguns concelhos do País. Não têm direito a títulos na imprensa que frequentemente veicula acriticamente dados oficiais globais que escondem profundas desigualdades («USF deram médicos de família a mais de dois milhões de pessoas» in Público 27.05.09). Numa nota de rodapé do Plano Nacional de Saúde 2004-2010, estimava-se em 1 milhão as pessoas em lista de espera para atribuição de MF. ${ }^{5}$ Estes dados reportam-se a 2004, mas a situação actual é certamente mais grave. Segundo um responsável do Ministério da Saúde, faltam actualmente, em Portugal, entre 300 a 400 MF. $^{6}$ Apesar de Portugal estar acima da média da OCDE em termos de rácio médico/1000 habitantes (3,5 clínicos em Portugal e 3,1 na OCDE), não tem havido uma gestão de recursos técnicos e humanos que permitisse evitar a grave situação a que chegaram algumas unidades de Cuidados de Saúde Primários (CSP). De facto o número de MF tem vindo a diminuir progressivamente na última década. O rá- 
cio MF por 100000 habitantes baixou de 54 para 51,5 entre 2002 e $2010 .^{2,4,5}$

A desigualdade no acesso aos CSP é, deste modo, mais outra vertente da desigualdade social e pouco tem sido feito para a minorar. Frequentemente, assistimos a uma afectação privilegiada de recursos materiais e humanos a determinados projectos, sem acautelar o agravamento dos cuidados noutros sectores. As Unidades de Saúde Familiar (USF) são um exemplo dum programa de indiscutível mérito, mas, nalgumas zonas do país, nomeadamente na área da Grande Lisboa, a sua criação deteriorou as condições de atendimento dos utentes «residuais» (utentes dos médicos que não quiseram ou não puderam aderir às USF e, sobretudo, utentes que já não tinham médico de família). Apesar de serem uma peça importante na melhoria dos CSP, as USF não vão dar resposta a curto prazo à falta de MF. $O$ mesmo se poderá dizer da contratação de médicos estrangeiros, que tem sido pontual a nível dos CSP, ou dos licenciados pelos novos cursos de Medicina que levarão cerca de 8 a 9 anos até começar a exercer. Impõe-se, urgentemente, outro tipo de medidas para resolver a situação dos utentes sem MF.

\section{UTENTES SEM MÉDICO - DIMENSÃO DO PROBLEMA}

Não é fácil quantificar e caracterizar os utentes sem médico, bem como o número exacto de MF a exercer num determinado momento. Portugal não dispõe de um sistema de informação que de forma exaustiva e atempada satisfaça as necessidades do país. ${ }^{2} \mathrm{~A}$ diferença entre os dados oficiais e os valores reais chega a atingir, nalgumas unidades de saúde, um factor de 2 (100\%). Médicos já reformados, transferidos para USF ou médicos que abandonaram o sector público aparecem por vezes nas estatísticas, que não são regularmente actualizadas.

Em 2004, a Secção Regional do Sul da Ordem dos Médicos publicou o Relatório da Comissão de Avaliação das Condições Técnicas para o exercício da Actividade dos Médicos nos Centros de Saúde da Administração Regional de Saúde de Lisboa e Vale do Tejo (ARS LVT). Este estudo resultava duma auditoria a 24 Centros de Saúde (CS) realizada entre 2002 e 2003 e verificava que $13 \%$ dos inscritos (110 mil de utentes) não tinham médico de família. Extrapolando os dados para a totalidade dos CS desta Administração de Saúde, o número de utentes sem médico atingiria os $392 \mathrm{mil}^{7} \mathrm{Num}$ inquérito sobre recém-nascidos de 7 CS dos concelhos de Amadora e Sintra, realizado entre 2005 e 2006, 24,9\% não tinham médico atribuído. ${ }^{8}$ Actualmente existem Unidades de CSP no concelho da Amadora com mais de $50 \%$ dos utentes sem médico.

O problema dos utentes sem MF, nos concelhos em que a situação é mais grave, deriva essencialmente do grande aumento demográfico e da má gestão dos recursos humanos. Nos concelhos de Amadora e Sintra, por exemplo, a população quase duplicou nas últimas décadas. No concelho de Sintra o aumento foi de cerca de $40 \%$ só na década entre 1991 e $2001 .^{8}$ Este crescimento demográfico não foi acompanhado pela expansão dos Cuidados de Saúde, quer Primários quer Secundários. O Hospital Fernando Fonseca, que serve as populações dos concelhos de Amadora e Sintra, presta actualmente cuidados a uma população cerca de duas vezes superior à inicialmente prevista. Por outro lado, o número de médicos nos CS tem vindo a decrescer progressivamente desde 1978. A falta de motivação, o sentimento de desvalorização, a desigualdade remuneratória e a insegurança laboral, têm levado muitos profissionais a abandonar o sector público para o privado, à opção pelo duplo emprego e a reforma antecipada. $^{2}$

Consequentemente, as condições de atendimento dos utentes sem médico têm-se agravado dramaticamente. As filas de espera de madrugada são um flagelo que tem vindo a aumentar nalguns CS. Raramente existem critérios de prioridade no atendimento destes utentes e só consegue consulta quem chegar mais cedo, o que não tem forçosamente relação com a gravidade da sua situação clínica. Aspectos básicos, como renovação de receituário para situações crónicas, só são assegurados parcialmente, motivando agudizações de patologias e descompensações perfeitamente evitáveis.

Os idosos são sem dúvida os utentes mais vulneráveis, dada a prevalência de doenças crónicas e de elevado nível de pobreza. Cerca de $63 \%$ dos idosos com idade igual ou superior a 75 anos são pobres, o que se repercute negativamente no acesso aos serviços de saúde. ${ }^{9}$ Por outro lado, não conseguem resolver facilmente os seus problemas com o recurso aos Serviços de Urgência, que vai sendo o escape dos utentes mais novos, 
com maior prevalência de situações agudas. A questão dos domicílios é particularmente grave. Os doentes acamados ou semi-acamados sem médico de família raramente conseguem uma consulta domiciliária.

A vigilância de grávidas e recém-nascidos tem também vindo a deteriorar-se, sobretudo na população emigrante. A proporção de emigrantes nos concelhos da Amadora e Sintra é das mais elevadas do país. Um estudo sobre factores de risco e morbilidade e mortalidade das crianças nos primeiros meses de vida nestes dois concelhos, realizado entre 2005 e 2006, revelou que os imigrantes apresentam maior vulnerabilidade, revelando valores significativamente mais elevados de desemprego, maior percentagem de gravidezes não vigiadas e de patologia materna, nomeadamente infecciosa, maior percentagem de risco social e maior mortalidade perinatal dos seus filhos. ${ }^{8}$

Os utentes «esporádicos» também não são contabilizados nas estatísticas oficiais de utentes sem médico. No entanto, o seu número é significativo na área da Grande Lisboa dado incluir os emigrantes sem residência permanente mas que, em muitos casos, são utilizadores regulares do CS durante vários anos. Não é fácil fazer uma caracterização mais precisa destes utentes, para além do número total, que pode atingir $8 \%$ dos inscritos numa unidade de saúde.

\section{O EXEMPLO INGLÊS}

A desigualdade no acesso à saúde não é, obviamente, um exclusivo português. No Reino Unido, outro país da UE com elevada desigualdade de rendimentos e social, criou-se uma diferença na prestação e na qualidade dos serviços de saúde a que eles chamaram de «lotaria do código postal» (postcode lottery ou "the huge gap between the best and the rest»). Se se vive na localidade errada, ou, em casos extremos, no lado errado da rua, pode obter-se um serviço de qualidade inferior ao do vizinho, ou mesmo não obter determinado serviço. ${ }^{10}$

A desigualdade dos serviços de saúde nasceu do facto de muitas decisões, nomeadamente sobre tratamentos e serviços disponibilizados, serem feitas localmente, mas teve o seu expoente nas fundholdings, sistema de gestão de Centros de Saúde criado nos anos 90 pelo governo conservador, que desenvolveu a noção de mercado interno. Os utentes dos CS organizados como fundholding tinham acesso mais rápido aos cuidados hospitalares que os dos CS convencionais, criando-se o «SNS a duas velocidades». Em 1997, o governo trabalhista substituiu as fundholdings por primary care groups e primary care trusts, mas manteve os princípios de mercado e muitas das desigualdades persistiram..$^{10,11}$

Infelizmente os ingleses têm sido particularmente bem sucedidos a exportar a sua ideologia de regras de mercado nos serviços públicos, causando erosão de valores fundamentais, como seja a igualdade no acesso aos serviços, e aumentando os custos. Ainda recentemente, o Dr John Reid, um ex-ministro da saúde trabalhista, defensor de modelos de saúde norte-americanos, esteve várias vezes em Portugal a defender a «liberdade de escolha» dos utentes (leia-se entre sector público e privado, através de contratos com o sector privado). Esta «liberdade» tem sido onerosa para os cidadãos britânicos, não só a nível da saúde, mas também da educação, onde tem aumentado os custos e a desigualdade. ${ }^{12,13}$ No nosso Plano Nacional de Saúde 2004-2010, já aparece também referida a necessidade de aumentar as "opções de escolha do cidadão». ${ }^{5}$

Outro problema enfrentado pelo Reino Unido tem sido a falta de recursos humanos, nomeadamente médicos e enfermeiros, motivada em parte pela falta de investimento governamental na sua formação.

A contratação de clínicos alemães para suprir as falhas nos Serviços de Urgência ao fim-de-semana causou alguma polémica, dados os elevadíssimos salários pagos, mas resolveu alguns problemas pontuais. O mesmo não aconteceu em relação à falta de MF. A expectativa de atrair 5000 MF espanhóis, numa altura em que havia grande desemprego médico em Espanha, ficou muito aquém das expectativas.

Maior sucesso houve na contratação de enfermeiras, nomeadamente das Filipinas e África do Sul, ainda que com custos pesados para os países de origem. Nelson Mandela chegou mesmo a apelar ao governo britânico para suspender esta contratação dado o impacto negativo para as populações locais. $\mathrm{O}$ pedido foi apenas parcialmente satisfeito.

\section{AS REFORMAS NOS CSP EM PORTUGAL}

Portugal tem obtido melhoria muito significativa e sustentada nos resultados em saúde desde o início da década de 70, conseguindo diminuir o grande atraso que nos separava dos outros países europeus. Nalguns in- 
dicadores, como a mortalidade infantil, conseguimos mesmo destacar-nos entre os países com melhores resultados.

Apesar desta melhoria, tem havido sucessivas críticas em vários relatórios de organismos internacionais, como a OCDE e a OMS, de baixos níveis de desempenho a nível da saúde tendo em conta os recursos utilizados, o que pode ser indicativo de uma distorcida afectação de recursos e de uma relativa falta de eficiência. O sistema de saúde português tem baixo desempenho em termos de equidade, eficiência, responsabilização e capacidade de resposta. ${ }^{2,5}$ A nível nacional, o Observatório Português de Sistemas de Saúde tem também vindo a criticar nos seus relatórios periódicos a existência de um défice de coordenação das medidas estruturantes e a implementação de uma política fragmentada, sem ter consideração pelos doentes. Como é referido no relatório da Primavera de 2008, «a prática governativa da saúde, em geral, mantém-se ocupada na resolução de problemas pontuais, com resposta à reacção dos profissionais, dos media, ou dos cidadãos utilizadores do sistema (estes menos, dado a resignação dominante), não criando medidas que possam prevenir estas e outras situações». A ausência de uma política adequada de recursos humanos tem também sido criticada pelas graves consequências para a evolução da saúde em Portugal. ${ }^{14}$

É nos CS que se tem agravado mais a falta não só de médicos, como de enfermeiros. As reformas antecipadas, o abandono para o sector privado e os atrasos nos concursos para recrutamento, têm levado a uma sangria de recursos vitais. O Estado tem sido incapaz de fixar os novos licenciados apesar dos elevados gastos que teve na sua formação. Esta é uma questão que se tem levantado também noutras profissões com formação longa e dispendiosa, como na aviação, onde se tem discutido a obrigatoriedade de trabalhar durante um determinado número de anos no sector público, antes de poder optar pelo sector privado, ou, alternativamente, indemnizar o Estado.

Em 2005 foi criada a Missão para os Cuidados de Saúde Primários, tendo como principais objectivos a melhoria da acessibilidade, qualidade, continuidade e eficiência dos cuidados de saúde, bem com o aumento da satisfação dos profissionais e utilizadores. O papel da Missão tem sido a implementação das USF e da rede de Cuidados Continuados nos CSP e a reforma da organização dos Serviços de Saúde Pública. Em Janeiro de 2010 havia 230 USF em funcionamento, abrangendo 2894983 utentes. O ratio utente/médico era de $1764 .{ }^{15}$ Houve ganhos indiscutíveis em termos de qualidade de cuidados prestados e satisfação dos profissionais e utilizadores nas USF, mas votou-se ao abandono o resto das unidades de saúde. Apesar de se referir um ganho de cobertura de $13.66 \%,{ }^{2}$ não é contabilizado que os médicos que integraram as USF deixaram de fazer atendimento aos utentes sem médico. De facto, a maioria dos médicos na área da Grande Lisboa disponibiliza pelo menos 3 a 4 horas do seu horário para os utentes sem MF, horas estas que deixam de ser realizadas quando os médicos integram as USF. Deste modo os ganhos de cobertura referidos são anulados pela diminuição do número de horas de consulta disponível para os utentes sem MF. Infelizmente, continua a incentivar-se a abertura de USF em locais em que se sabe que isso vai agravar dramaticamente a situação dos utentes sem MF.

A criação dos Agrupamentos de CS (ACES) também não foi capaz de dar resposta às graves situações deixadas pela saída de médicos e enfermeiros para as USF ou para o sector privado. $\mathrm{O}$ atraso na regulamentação destas estruturas e a sua falta de autonomia tem perpetuado situações graves no funcionamento de várias Unidades de Cuidados Saúde Personalizados (ex-CS ou suas extensões).

Parecem, deste modo, repetir-se alguns dos erros das reformas do sistema inglês quando criaram as fundholdings-afectação prioritária de recursos a determinados projectos, mas agravando a situação dos restantes CS, aumentando a desigualdade no acesso aos cuidados de saúde e criando o já referido SNS a duas velocidades.

\section{MUDANÇA DE PARADIGMA}

Um passo fundamental para resolver a actual situação de falta de MF poderá passar pela alteração do paradigma dos CSP centrados no médico de família e reconhecer, como o fizeram vários países europeus, que os enfermeiros são fundamentais para a resolução do problema da falta de médicos e estão grandemente subaproveitados. Por outro lado, ao contrário dos MF, não há falta de enfermeiros em Portugal. Há, isso sim, muitos enfermeiros no desemprego. 
Países como a Holanda, a Dinamarca e o Reino Unido, têm vindo a transferir para os enfermeiros numerosas tarefas sem decréscimo de qualidade dos cuidados de Saúde, o que permitiu aumentar a dimensão dos seus ficheiros para cerca de 2200 a 2300 utentes. ${ }^{16}$ Uma parte significativa da prevenção e da vigilância de doenças crónicas é feita, nesses países, pelos enfermeiros. No Reino Unido, os enfermeiros fazem numerosas consultas, a que chamam «Clinics», algumas delas sozinhos, outras em colaboração com o médico de família. São exemplo a «consulta» de Saúde do Viajante (Travel Advice and Immunisation Clinic, feita por uma enfermeira com formação nesta área), a vigilância de Diabéticos (Diabetic Clinic - ensino, verificação do controlo metabólico e avaliação podológica, consulta esta frequentemente realizada em conjunto com um médico, ou intercalada nas consultas médicas), a vigilância de hipertensos (High Blood Pressure Clinic), cessação tabágica (Stop Smoking Clinic), os exames de rotina (Well Women / Well Man Clinic - discussão de estilos de vida saudáveis, avaliação de TA, aconselhamento sobre rastreios), os exames aos idosos (Health Screening for the Elderly-consulta anual pela enfermeira para utentes $\geq$ 75 anos), as colpocitologias de rastreio (realizadas pela enfermeira), o aconselhamento na menopausa (Menopause Clinic), a vigilância de asmáticos (Asthma Clinic, em que uma enfermeira com formação específica verifica a técnica de uso de inaladores, faz espirometrias, avalia o grau de controlo e faz aconselhamento), a consulta de Saúde Infantil (com consultas feitas pelo MF e enfermeira e outras só pela enfermeira, ou, pela Health Visitor), a consulta de Saúde Materna (frequentemente em alternância entre a Midwife e o médico). Nem todas estas consultas existem simultaneamente em cada CS, dependendo da disponibilidade de enfermeiros com formação nessas áreas, mas todas elas obedecem a protocolos acordados internamente. Deste modo, reduz-se o número de consultas médicas por utente inscrito, permitindo aumentar substancialmente a dimensão dos ficheiros sem acréscimo relevante da carga de trabalho e mantendo a qualidade na prestação de cuidados.

\section{CONCLUSÃO}

Portugal precisa urgentemente de reduzir as assimetrias na acessibilidade aos Cuidados de Saúde Primá- rios. As reformas dos CSP têm-se centrado na implementação das USF, mas os ganhos de cobertura com as USF são anulados pelo agravamento da assistência aos utentes sem MF. O modelo dos ficheiros de 1550 utentes (ou mesmo 1760, nas USF) está há muito ultrapassado. Impõem-se alterações corajosas à organização dos CSP, melhorando a sua eficiência, o que deveria passar pela valorização do papel dos enfermeiros, permitindo um aumento significativo (na ordem de 20 a 35\%) da dimensão das listas dos médicos de família.

\section{REFERENCIAS BIBLIOGRÁFICAS}

1. Niehues J, Income Inequality within and between European Countries. European User Conference for EU-LFS and EU-SILC. Mannheim, 06/03/2009.

2. Biscaia A, Martins J, Carreira M, Gonçalves I, Antunes A, Ferrinho P. Cuidados de Saúde Primários em Portugal - reformar para novos sucessos. $2^{\mathrm{a}}$ ed. Lisboa: Fundação AstraZeneca; 2008.

3. Simões J. Notícias da Saúde. Público, 11/01/2010.

4. OCDE. Health at a Glance 2009. OECD Indicators. Paris: OECD Publishing; 2009.

5. Direcção Geral da Saúde. Plano Nacional de Saúde 2004-2010. Disponível em http://www.dgs.pt/upload/membro.id/ficheiros/ i006756.pdf e http://www.dgs.pt/upload/membro.id/ficheiros/ i006757.pdf [acedido em 23/02/2010].

6. Campos A. Ministério revela que faltam entre 300 e 400 médicos de família e em 2013 poderá ser pior. Público, 16/12/2010.

7. Secção Regional do Sul da Ordem dos Médicos. Relatório da Comissão de Avaliação das Condições Técnicas para o Exercício da Actividade dos Médicos nos Centros de Saúde da Administração Regional de Saúde de Lisboa e Vale do Tejo. Lisboa: Ordem dos Médicos; 2004.

8. Machado MC, Santana P, Carreiro MH, Nogueira H, Barroso MR, Dias A. Iguais ou diferentes? Cuidados de saúde materno-infantil a uma população de imigrantes. Prémio Bial de Medicina Clínica 2006. Porto: Bial; 2006.

9. Costa AB. Um olhar sobre a pobreza: vulnerabilidade e exclusão social no Portugal contemporâneo. Lisboa: Gradiva; 2008.

10. Butler P. Q\&A: Postcode lottery. Disponível em: http://www.guardian.co. uk/society/2000/nov/09/NHS [acedido em 23/02/2010]..

11. Pollock AM. NHS Plc: the privatisation of our health Care. London: Verso Books; 2005.

12. Press Association. Education reforms risk widening social gap. Disponível em: http://www.guardian.co.uk/education/2006/feb/22/schools.uk4 [acedido em 23/02/2010].

13. Crouch C. Commercialization or citizenship: education policy and the future of public services. London: Fabian Society; 2003.

14. Observatório Português de Sistemas de Saúde. Relatório Primavera 2008. Lisboa: Escola Nacional de Saúde Pública; 2008.

15. Missão para os Cuidados de Saúde Primários. Disponível em: http://www.mcsp.min-saude.pt [acedido em 23/02/2010].

16. Conferência "Organização da Prestação de Cuidados de Saúde Primários". Cascais; 25/06/2008. 
ENDEREÇO PARA CORRESPONDÊNCIA

Isabel Cortez

Rua Pedrouços, $77-1 .^{\circ}$ Dto.

1400-286 LISBOA

E-mail: icortez@doctors.org.uk

Recebido em 26/02/2010

Aceite para publicação em 26/03/2010

\section{ABSTRACT}

\section{DISPARITY IN THE ACCESS TO PRIMARY HEALTH CARE: THE HIDDEN FACET OF THE NEW SURGERIES}

Income and social inequality are very high in Portugal. Variation in access to primary care services contributes to inequality and no effective policies have improved these results. Resource allocation has often aggravated inequality in access to health care, as did some of the NHS reforms in the UK in the nineties.

There are over a million patients without a GP in Portugal, $40 \%$ located in the area of Administração Regional de Saúde de Lisboa e Vale do Tejo. Neither the new Primary Care Surgeries, nor the recruitment of foreign doctors, nor yet the new Medical Courses will help, in the short term, to solve this problem.

There is an urgent need for better solutions. A few EU countries solved the problem of shortage of GPs by contracting more nurses and transferring to them some areas of prevention and surveillance of chronic illnesses. GPs have now bigger lists of patients but work burden has not increased substantially and quality of care has been kept.

Keywords: Inequality; Access; Primary Care. 